\title{
Interação Entre Domínio Motor Amplo e de Linguagem no Processo de Desenvolvimento Típico: Uma Revisão Integrativa
}

\author{
Letícia Hermes ${ }^{1}$, Simone Nicolini de Simoni ${ }^{2}$, \\ Angela Regina Maciel Weinmann ${ }^{3}$, Marcia Keske-Soares ${ }^{4}$
}

\begin{abstract}
RESUMO
O desenvolvimento infantil constrói-se sobre pilares fundamentais, também denominados domínios, entre eles o motor e a linguagem, que emergem gradualmente de complexas cascatas de interações biológicas e físicas. O objetivo dessa revisão integrativa de literatura foi sintetizar estudos que avaliaram algum processo de interação entre componentes do desenvolvimento motor amplo e da linguagem oral, especialmente a aquisição das primeiras produções de fala, em um processo de desenvolvimento típico em crianças de zero a 36 meses de idade. Mediante uma busca sistematizada da literatura, seis estudos publicados entre 2001 e 2015 foram integrados sinteticamente em uma análise crítica por pares. Os estudos selecionados não oferecem um consenso que, provavelmente, pode ser explicado pela heterogeneidade metodológica, especialmente marcada pelo restrito número amostral, diversidade de objetivos, instrumentos e métodos de análise, notadamente marcada por a variável de desfecho ser centrada em escores gerais do domínio, ou marcos pontuais. Estes últimos parecem ser mais específicos em sinalizar o componente de interação que se reflete no domínio como um todo. Nesse complexo processo interacional, o desenvolvimento motor parece preparar terreno para o desenvolvimento da linguagem oral, especialmente nas primeiras produções de fala, favorecendo uma maior exploração do ambiente e gerando maior resposta comunicativa, especialmente de pais e cuidadores. Sugerem-se novos estudos que comparem marcos de desenvolvimento num paralelo entre os domínios, com delineamento longitudinal e análise multivariada, a fim de aproximar-se da relação antecedente-consequente que melhor explicaria esse processo de interação, o qual ainda não está completamente elucidado.
\end{abstract}

Palavras-chave: Desenvolvimento infantil. Desenvolvimento da linguagem. Destreza motora.

INTERACTION BETWEEN GROSS MOTOR AND LANGUAGE DOMAIN IN TYPICAL DEVELOPMENT PROCESS: AN INTEGRATIVE REVIEW

\section{ABSTRACT}

Child development is built on fundamental pillars, also called domains, including motor and language, which gradually emerge from complex cascades of biological and physical interactions. The main of this integrative literature review was to synthesize studies that evaluated some interaction process between gross motor development and oral language components, especially the acquisition of the first speech sounds productions, in a typical developmental process in children from zero to 36 months of age. Through a systematic literature search, six studies published between 2001 and 2015 were synthetically integrated into a critical review. The selected studies do not offer a consensus that can probably be explained by the methodological heterogeneity, especially marked by the limited sample number, diversity of objectives, instruments and methods of analysis, notably marked by the outcome variable being centred on general domain scores, or milestones. punctual. The last one mentioned seems to be more specific in flagging the interaction component that is reflected in the domain as a whole. In this complex interactional process, motor development seems to pave the way for the development of oral language, especially in the first speech productions, favouring a greater exploration of the environment and generating a greater communicative response, especially from parents and caregivers. We suggest further studies that compare developmental milestones in a parallel between the domains, with longitudinal delineation and multivariate analysis, in order to approach the antecedent-consequent relationship that would best explain this interaction process, which is not yet fully elucidated.

Keywords: Child development. Language development. Motor skills.

RECEBIDO EM: 13/12/2019

MODIFICAÇÕES SOLICITADAS EM: 20/3/2020

ACEITO EM: 6/4/2020

\footnotetext{
Fisioterapeuta. Doutoranda do Programa de Pós-Graduação em Distúrbios da Comunicação Humana da Universidade Federal de Santa Maria (UFSM). http:// lattes.cnpq.br/8082653072615230. https://orcid.org/0000-0003-0331-6345. letihermes1@gmail.com

Fonoaudióloga. Doutoranda no Programa de Pós-Graduação em Distúrbios da Comunicação Humana da Universidade Federal de Santa Maria (UFSM). http:// lattes.cnpq.br/7039894531418318. https://orcid.org/0000-0002-3764-4171. simonedesimoni@hotmail.com

3 Neonatologista. Pós-doutorado no The Hospital for Sick Children, University of Toronto, Canadá. Professora do Curso de Medicina da Universidade Franciscana (UFN). Coordenadora da Residência Médica - Coreme. Professora titular da Universidade Federal de Santa Maria (UFSM) (professora colaboradora). http:// lattes.cnpq.br/9151119377173425. https://orcid.org/0000-0003-2381-9396. noca.rm@yahoo.com

${ }^{4}$ Fonoaudióloga. Doutora em Linguística Aplicada pela Pontifícia Universidade Católica do Rio Grande do Sul (PUCRS). Professora titular do Curso de Fonoaudiologia e do Programa de Pós-Graduação em Distúrbios da Comunicação Humana da Universidade Federal de Santa Maria (UFSM). http://lattes. cnpq.br/2993790524055307. https://orcid.org/0000-0002-5678-8429. marcia-keske.soares@ufsm.br
} 


\section{INTRODUÇÃO}

Desde o nascimento, a criança vivencia um processo contínuo e sequencial de aquisição de capacidades que ocorre com o avanço da idade, provocadas pela interação entre as exigências da tarefa, a biologia do indivíduo e as condições ambientais (MEDINA ALVA et al., 2015). O desenvolvimento típico envolve um processo gradual de aquisições alicerçadas na maturação do Sistema Nervoso Central, especialmente sensível a uma ampla gama de experiências, mostrando uma capacidade notável de alterações plásticas que influenciam os resultados comportamentais ao longo da vida (KOLB; HARKER; GIBB, 2017; ZAGO et al., 2017)

$O$ desenvolvimento infantil constrói-se sobre pilares fundamentais, também denominados domínios, os quais integram funções motoras, psicossociais, linguísticas e cognitivas (MEDINA ALVA et al., 2015). A interação entre esses domínios vem sendo explicada pelo fato de que as habilidades emergentes em um deles podem ter reflexos indiretos para o desenvolvimento e a experiência em outros, emergindo gradualmente de complexas cascatas de interações biológicas e físicas, fenômeno conceitual denominado "cascatas de desenvolvimento" (D'SOUZA; D'SOUZA; KARMILOFF-SMITH, 2017; IVERSON, 2018).

Entre os domínios que podem apresentar interação em um processo de desenvolvimento típico, serão enfocados os domínios motor e de linguagem. Essa interação vem sendo abordada em alguns estudos (CHOO et al., 2016; FREZZATO et al., 2017; HODGSON; HUDSON, 2018; IVERSON, 2010, 2018; LIU; ZAHRT; SIMMS, 2018).

Uma eventual associação entre esses dois domínios poderia sugerir um processo de interação entre as suas aquisições, denominadas marcos de desenvolvimento, com ênfase à linguagem oral (especialmente produção da fala), enquanto componente do campo de estudo da linguagem e motor amplo como componente do domínio motor. Estas habilidades podem ser precursoras, catalisadoras e, quando relacionadas ou coemergentes, podem ser preditoras ou estarem integradas no processo de aquisição típico (IVERSON, 2018).

Apesar de outros estudos já terem feito associação entre esses dois domínios, muitos ainda o fazem com vistas à coocorrência de comprometimentos motores e de linguagem com alguma condição atípica envolvida (CHOO et al., 2016; FREZZATO et al., 2017; LIU; ZAHRT; SIMMS, 2018).

A associação entre domínio motor e de linguagem, de modo abrangente, foi discutida em uma revisão de Iverson (2010). Esse artigo, no entanto, desti- nou-se a descrever as teorias publicadas até então, ao invés de sintetizar os estudos que contemplavam esse processo de interação. Após a referida publicação, avançaram-se nos estudos nessa área e o enfoque foi sendo modificado. Nesse ínterim, foram emergindo estudos que passaram a analisar tarefas motoras mais amplas, convergindo para a adequação de uma revisão integrativa da pesquisa, alvo do presente estudo.

Por outro lado, componentes relacionados à interação mão e boca já parecem ter relação claramente estabelecida na literatura (VAINIO, 2019). Aparentemente essas habilidades são fundamentadas por conexões anatômicas e funcionais que permeiam uma base neuronal comum (HODGSON; HUDSON, 2018). Esta interação específica, já suscitada por teorias pregressas, foi reforçada especialmente sobre unidades de fala nas quais a linguagem humana é construída, já estando estabelecida no período neonatal e tendo influência direta sobre o desenvolvimento da fala (VAINIO, 2019). O fato de a relação entre funções de mão e boca estarem bem estabelecidas, desperta o interesse para abranger o olhar sobre outras habilidades motoras envolvidas nesse processo.

Sabe-se ainda muito pouco sobre o desenvolvimento cerebral dos bebês em relação à linguagem e, menos ainda, sobre a conexão entre os sistemas motor e de linguagem em desenvolvimento (NELSON; CAMPBELL; MICHEL, 2014). Diante do exposto, busca-se responder se o desenvolvimento de ambos os domínios - linguagem, nesse caso, o processo de aquisição da fala, e o motor, compreendendo atividades motoras amplas - também estariam relacionados em um processo de interação. Pressupõem-se que haja interação, porém carecem de subsídios teóricos que possam fundamentar essa hipótese. Dada a confirmação, abre-se caminho para posterior avaliação de marcos coemergentes e eventuais associações no processo de desenvolvimento típico de aquisições.

Assim, o presente estudo objetivou identificar e sintetizar estudos observacionais que avaliaram ou descreveram processo de interação entre componentes do desenvolvimento motor amplo e da linguagem, especialmente do componente oral, relacionado à produção da fala no processo de desenvolvimento típico de crianças de zero aos 36 meses de idade.

\section{METODOLOGIA}

O presente estudo foi delineado enquanto revisão integrativa da literatura, em que a busca nas bases de dados ocorreu no período de setembro a outubro de 2019. Essa busca foi conduzida em bases 
eletrônicas e de forma manual a partir das referências de estudos afins. O método da revisão será descrito de acordo com as etapas destinadas à revisão, sejam elas: Identificação, Screening, Elegibilidade e Inclusão dos artigos e resultados.

\section{Identificação}

Após essa definição da questão de pesquisa, foram escolhidas três bases de dados pela relevância que possuem na área científica e de saúde, sendo elas: PubMed, Scopus e Web of Science.

Foram elaborados construtos a fim de delinear a pesquisa e a busca pelos artigos. Os construtos foram pensados em relação aos seguintes critérios: a) Construto relacionado à criança: child $O R$ infant $O R$ early; b) Construto relacionado ao desenvolvimento global: development $O R$ acquisition OR skills OR abilit*; c) Construto relacionado à linguagem/fala: speech OR language OR phonolog* OR speech-sound $O R$ articulat; d) Construto relacionado ao movimento/ motor: movement $O R$ motor $O R$ fine-motor $O R$ coordination; e) Construto relacionado à normalidade: typic* $O R$ normal; f) Construto patologia (realizado para exclusão com o booleano AND NOT) disturb* $O R$ disord* OR difficult* OR syndrom*; g) Construto adulto (para exclusão): adult.

Os construtos incluíram palavras de relevância e frequência presentes nas bases de dados. Utilizou o $\left({ }^{*}\right)$ "wildcard character" a fim de abranger as derivações das palavras destacadas. Além disso, usou-se também o hífen (-) a fim de buscar exatamente o termo escolhido. Os construtos foram pesquisados separadamente, selecionados com o filtro de Title/Abstract nas bases PubMed e Scopus e apenas com Title na base Web of Science, e, finalmente, foram integrados com os booleanos, caracterizando-se a seguinte sequência de pesquisa:

\section{(a) AND (b) AND (c) AND (d) AND (e) AND NOT (f) AND NOT (g)}

Os filtros utilizados nas três bases de dados limitaram a busca de acordo com a língua, optando-se por inglês, espanhol e português, a seres humanos e à idade-alvo. No critério relacionado à sequência de período das pesquisas, optou-se por não delimitar o tempo de estudo, abrangendo todas as décadas possíveis, assim como não se limitou o acesso a esses artigos.

Ainda na fase de identificação, algumas características, de acordo com as bases de dados, merecem devida descrição. Nas três bases de dados foi possível delinear a idade-alvo da população dos artigos, selecionando-se as idades inferiores a quatro anos dis- poníveis nas plataformas. Apenas na base dados da Scopus foi possível selecionar a área abrangente das revistas, escolhendo-se, então, revistas destinadas ao trabalho multidisciplinar e às ciências da saúde. $\mathrm{Na}$ base de dados da Web Of Science e PubMed foi possível optar pelo filtro de "humanos", excluindo-se pesquisas relacionadas a animais.

\section{Screening}

A segunda etapa da revisão integrativa foi definida como screening, momento em que, separadamente, duas pesquisadoras realizaram a busca com os critérios descritos na identificação após a exclusão das duplicatas. Os critérios estabelecidos para a escoIha dos titles e abstracts foram estudos realizados na idade desejada, ou seja, de zero até 36 meses, bem como relação direta com o questionamento de pesquisa, em que ambos os critérios relacionados com os marcos de desenvolvimento motor e de linguagem/ fala estivessem presentes na descrição. Foram excluídos: artigos que envolviam adaptação ou validação de testes; aqueles cujo delineamento metodológico ou análise tivessem sido conduzidos sob a ótica de alguma exposição, condições especiais ou patologias/distúrbios; que focassem a relação de componentes que envolviam relação mão e boca, já bem-suportada pela extensa literatura; e, ainda, os que não descrevessem interação entre os domínios-alvo.

Cada pesquisadora elaborou uma tabela com os titles e abstracts selecionados para posterior conferência, em caso de dúvida entre as duas, ou de divergência na aplicação dos critérios, uma terceira juíza seria solicitada. Além disso, os artigos selecionados nessa fase, tiveram suas referências revisadas para possíveis inclusões por outras fontes.

\section{Elegibilidade e inclusão dos artigos}

Realizada a comparação do screening entre as pesquisadoras, foi feita a leitura dos artigos na íntegra para verificar se havia relação direta com o objetivo da revisão. Os aspectos que permearam a inclusão final dos estudos serão descritos nos resultados.

\section{RESULTADOS E DISCUSSÃO}

Os resultados serão apresentados e discutidos na sequência de Figura e Tabela a seguir.

A Figura 1 apresenta o fluxograma da revisão, ou seja, o número total de artigos encontrados nas bases de dados, bem como os critérios aplicados que culminaram nos artigos selecionados. 
Figura 1 - Fluxograma da Revisão

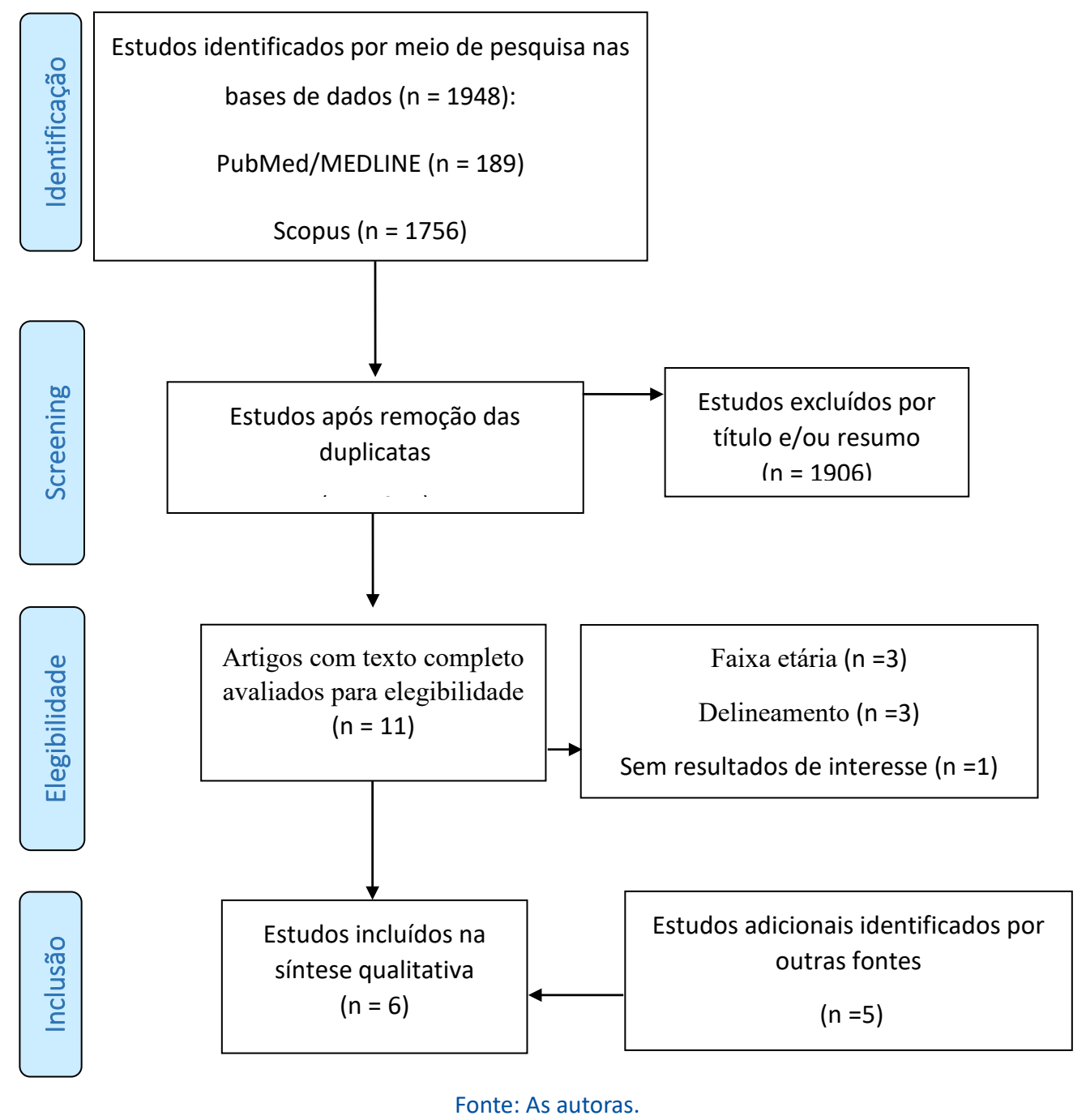

Conforme o fluxograma apresentado, observa-se que são poucos os estudos que atendem aos critérios estabelecidos na busca. $O$ foco principal foi a descrição de uma interação entre os domínios motor amplo e de linguagem/fala nos processos de aquisição em crianças típicas ou que não tenham sido expostas a qualquer tipo de condição ou alteração específica.

Um aspecto que envolveu a delimitação dos critérios e cabe ser discutido é a definição de alguns conceitos essenciais para a busca e a discussão de aspectos inerentes, em especial ao componente da linguagem, o qual não está bem estabelecido na literatura, sendo empregado para conceituar todos os seus múltiplos componentes. Esta envolve um construto muito amplo, que permeia todo o simbolismo da comunicação humana, seja ela palavra, sons, sinais ou comportamentos para receber e expressar ideias, pensamentos e sentimentos (LIU; ZAHRT; SIMMS, 2018).
O escopo deste estudo atém-se a discutir o processo de aquisição e desenvolvimento da fala enquanto desfecho da linguagem oral. Por isso, a limitação do escopo acabou compreendendo a exclusão da relação mão e boca, como componente já bem estabelecido na literatura, bem como não foi extensivo a todos os aspectos do desenvolvimento motor, atendo-se ao componente motor amplo. Essa definição também não é clara e a carência de uma definição padronizada nos termos utilizados por estudos afins torna a busca muito heterogênea, resultando na omissão de artigos que usam outros termos, mas essencialmente estão avaliando e descrevendo o mesmo conceito (LOGAN et al., 2018).

A partir dos artigos selecionados foram extraídas informações substanciais que possibilitassem caracterizar seu delineamento metodológico e os principais resultados acerca da interação entre os domínios. Os principais aspectos inerentes a essa fase da análise encontram-se descritos na Tabela 1. 
Tabela 1 - Características dos estudos selecionados na revisão

\begin{tabular}{|c|c|c|c|c|c|}
\hline Título / Autor (ano) & Objetivo & Delineamento & Amostra & Avaliação & Conclusões principais \\
\hline $\begin{array}{l}\text { Co-occurrence of preverbal } \\
\text { vocal behavior and motor } \\
\text { action im early infancy / } \\
\text { Ejiri \& Masataka, (2001) }\end{array}$ & $\begin{array}{l}\text { Avaliar a coocorrência de } \\
\text { comportamentos vocais e } \\
\text { ações motoras em lactentes }\end{array}$ & Longitudinal & $\begin{array}{l}\text { Quatro } \\
\text { crianças } \\
\text { japonesas de } 6 \\
\text { a } 11 \text { meses }\end{array}$ & $\begin{array}{l}\text { Observação do comportamento natural } \\
\text { da criança em interação com a mãe por } \\
90 \text { minutos (vídeo) }\end{array}$ & $\begin{array}{l}\text { Balbucio pode não ser um simples } \\
\text { fenômeno fonológico, mas estar } \\
\text { intimamente ligado a um } \\
\text { comportamento rímico generalizado. }\end{array}$ \\
\hline $\begin{array}{c}\text { Stability of serial } \\
\text { assessments of motor } \\
\text { and communication abilities } \\
\text { in typically } \\
\text { developing infants - } \\
\text { implications for screening / } \\
\text { Darrah, et. al. (2003) }\end{array}$ & $\begin{array}{l}\text { Avaliar a estabilidade dos } \\
\text { escores motores finos, motores } \\
\text { brutos e de comunicação dos } \\
\text { bebês }\end{array}$ & Longitudinal & $\begin{array}{l}102 \text { crianças } \\
\text { canadenses aos } \\
9,11,13,16 \mathrm{e} \\
21 \text { meses }\end{array}$ & $\begin{array}{c}\text { The Peabody Developmental Motor } \\
\text { Scales e Commenication Symbolic } \\
\text { Behavior } \\
\text { Scales-Developmental Proflie }\end{array}$ & $\begin{array}{c}\text { Motor fino, motor bruto e } \\
\text { comunicação habilidades parecem } \\
\text { desenvolver-se independentemente. }\end{array}$ \\
\hline $\begin{array}{l}\text { Individual differences in } \\
\text { language development: } \\
\text { relationship with motor skill } \\
\qquad \text { at } 21 \text { months / } \\
\text { Alcock \& Krawczyk, (2010) }\end{array}$ & $\begin{array}{l}\text { Avaliar a associação de uma } \\
\text { variedade de } \\
\text { habilidades motoras com o } \\
\text { desenvolvimento da } \\
\text { linguagem. }\end{array}$ & Transversal & $\begin{array}{l}129 \text { crianças } \\
\text { do Reino } \\
\text { Unido de } 21 \\
\text { meses }\end{array}$ & $\begin{array}{c}\text { McArthu-Bates Commuticative } \\
\text { Development Inventory; } \\
\text { Tests of motor control } \\
\text { Manual gesture; } \\
\text { Habilidade motora oral; } \\
\text { Gesto manual; } \\
\text { CDI- Wordt and Gestures; } \\
\text { Bayley Scales of Infant Development } \\
\text { (BSID-Motor) (Bayley, 1993); } \\
\text { Questionários aos pais (motor e } \\
\text { linguagem) }\end{array}$ & $\begin{array}{l}\text { Encontrou forte correlação entre } \\
\text { habilidades motoras orais e linguagem } \\
\text { produtiva sugerindo uma base } \\
\text { articulatória comum. Motor grosso } \\
\text { parece estar relacionado às desordens e } \\
\text { não ao típico, alvo desse estudo. }\end{array}$ \\
\hline $\begin{array}{l}\text { Attainment of sitting and } \\
\text { walking predicts } \\
\text { development of productive } \\
\text { vocabulary between ages } 16 \\
\text { and } 28 \text { months / } \\
\text { Oudgenoeg-Paz et al, (2012) }\end{array}$ & $\begin{array}{l}\text { Analisar as relações entre a } \\
\text { idade de sentar-se sem apoio e } \\
\text { caminhar independentemente } \\
\text { e desenvolvimento da } \\
\text { linguagem produtiva }\end{array}$ & Longitudinal & $\begin{array}{l}55 \text { crianças } \\
\text { holandesas de } \\
16 \text { a } 28 \text { meses }\end{array}$ & $\begin{array}{l}\text { Relatório dos pais (prévio à aquisição) } \\
\text { McArthur Communicative } \\
\text { Developmental } \\
\text { Inventories } \\
\text { (a cada quatro meses) }\end{array}$ & $\begin{array}{l}\text { Sentar-se sem apoio e caminhar } \\
\text { independentemente antes, mostram um } \\
\text { nivel geral mais alto } \\
\text { e maior taxa de crescimento do } \\
\text { vocabulário produtivo, } \\
\text { respectivamente }\end{array}$ \\
\hline $\begin{array}{l}\text { Infant Language } \\
\text { Development Is Related to } \\
\text { the Acquisition of Walking } \\
\text { (Estudo I) / } \\
\text { Walle \& Campos, (2014) }\end{array}$ & $\begin{array}{c}\text { Saber se o inicio da caminhada } \\
\text { está relacionado ao } \\
\text { desenvolvimento } \\
\text { linguagem infantil }\end{array}$ & Longitudinal & $\begin{array}{l}75 \text { crianças } \\
\text { Americanas de } \\
10 \text { a } 13,5 \\
\text { meses }\end{array}$ & $\begin{array}{l}\text { Questionário online marcos motores e } \\
\text { MacArthur-Bates Long Form } \\
\text { Vocabulary Checklist: Level I }\end{array}$ & $\begin{array}{l}\text { A aquisição da caminhada esteve } \\
\qquad \text { associada } \\
\text { com um aumento gignificativo na } \\
\text { linguagem receptiva e produtiva, } \\
\text { independentemente da idade }\end{array}$ \\
\hline $\begin{array}{l}\text { Infant Language } \\
\text { Development Is Related to } \\
\text { the Acquisition of Walking } \\
\text { (Estudo II) / } \\
\text { Walle \& Campos, (2014) }\end{array}$ & $\begin{array}{l}\text { Analisar as diferenças no } \\
\text { desenvolvimento da linguagem } \\
\text { infantil } \\
\text { entre grupos (engatinhar e } \\
\text { caminhar) }\end{array}$ & Transversal & $\begin{array}{l}44 \text { crianças } \\
\text { Americanas de } \\
12,5 \text { meses }\end{array}$ & $\begin{array}{l}\text { Observação do comportamento natural } \\
\text { das diades por } 90 \text { minutos (aspectos } \\
\text { sociais do ambiente) }\end{array}$ & $\begin{array}{l}\text { Variaçōes no ambiente social previram } \\
\text { diferencialmente o desenvolvimento da } \\
\text { linguagem de crianças que engatinham } \\
\text { e caminham. }\end{array}$ \\
\hline $\begin{array}{c}\text { A Cross-National } \\
\text { Investigation of the } \\
\text { Relationship Between Infant } \\
\text { Walking and Language } \\
\text { Development / } \\
\text { He, Walle \& Campos, (2015) }\end{array}$ & $\begin{array}{l}\text { Analisar componente } \\
\text { epigenético na relação do } \\
\text { inicio da locomoção com } \\
\text { desenvolvimento da linguagem }\end{array}$ & Transversal & $\begin{array}{l}40 \text { crianças } \\
\text { americanas e } \\
42 \text { crianças } \\
\text { chinesas de } \\
12,5 \text { meses }\end{array}$ & $\begin{array}{l}\text { MacArthur-Bates Long Form } \\
\text { Vocabulary Checklist: Level I }\end{array}$ & $\begin{array}{l}\text { Bebês que caminham apresentaram } \\
\text { maiores } \\
\text { vocabulários receptivos e produtivos } \\
\text { do que os que engatinham, na mesma } \\
\text { idade, independente da nacionalidade }\end{array}$ \\
\hline
\end{tabular}


Todos os estudos selecionados mostraram-se bastante heterogêneos em seus objetivos, delineamentos e variáveis analisadas, dificultando um agrupamento dos resultados e inviabilizando um processo de revisão mais robusto dos mesmos, limitando o delineamento metodológico, sendo a revisão integrativa considerada a ferramenta mais compatível ante o objetivo deste estudo.

Apesar de serem desenvolvidos estudos observacionais que descrevam o aparecimento de marcos de desenvolvimento em relação à cronologia da idade das crianças (ERTEM et al., 2018), ainda são escassos aqueles que analisem a interação que compõe a questão de pesquisa do presente estudo. Em contrapartida, evidências crescentes demonstram conexões anatômicas e funcionais entre os movimentos da boca e os movimentos manipulativos das mãos, sendo essa questão amplamente revisada em estudo recente publicado por Vainio (2019). Este autor enfatiza uma gama de habilidades manipulativas que podem facilitar o desenvolvimento de certos gestos articulatórios durante o desenvolvimento precoce da fala.

Cronologicamente, o primeiro estudo que passa a trazer um olhar sobre a movimentação ampla da criança, ainda que entre outros aspectos, como manipulação e preensão de objetos, foi desenvolvido por Ejiri e Masataka (2001). Estes autores avaliaram a coemergência de vocalizações e movimentos, sinalizando um predomínio de movimentos definidos como rítmicos (envolvendo pernas e/ou braços com repetição rítmica) no surgimento das primeiras vocalizações de 4 bebês japoneses acompanhados dos 6 aos 11 meses.

Outro aspecto relevante diz respeito à forma de análise dos estudos que se sucederam (ALCOCK; KRAWCZYK, 2010; DARRAH et al., 2003). Os referidos estudos traçavam um olhar voltado à análise dos escores dos testes empregados nas avaliações, bem como traziam muito presente a questão da relação com a destreza manual, dominância ou gesto simbólico de apontamento, fortemente relacionados ao desenvolvimento da linguagem, por vezes colocada de modo muito genérico, sem especificar o componente em questão. Os estudos posteriores (HE; WALLE; CAMPOS, 2015; OUDGENOEG-PAZ; VOLMAN; LESEMAN, 2012; WALLE; CAMPOS, 2014) passaram a direcionar uma análise mais voltada para a aquisição de marcos motores específicos com o desenvolvimento da linguagem oral, passando a encontrar relações nas aquisições desses marcos com vocabulário expressivo e produtivo.
Essa perspectiva de observação pareceu ser determinante nas conclusões das pesquisas analisadas, pois os primeiros estudos (ALCOCK; KRAWCZYK, 2010; DARRAH et al., 2003) que examinaram escores compostos das escalas completas, especialmente em termos motores, acabaram encontrando pouca ou nenhuma interação entre os domínios motor e linguagem. Os demais (HE; WALLE; CAMPOS, 2015; OUDGENOEG-PAZ; VOLMAN; LESEMAN, 2012; WALLE; CAMPOS, 2014), que direcionaram sua análise para a aquisição de marcos motores amplos, mais específicos, ditando uma possível interação do marco avaliado com a aquisição da fala enquanto componente da linguagem, culminaram em conclusões distintas de seus antecessores.

Essa divergência parece advir do fato de que escores contemplam aspectos amplos do processo de desenvolvimento, que podem não sinalizar situações pontuais de interação, como ocorreria em uma análise pautada em marcos. Por exemplo, em um escore que compreende uma série de marcos, a criança pode não apresentar um único marco específico e pontuar bem no somatório final.

Em relação aos delineamentos metodológicos, esses também mostraram-se muito diversos entre os estudos selecionados, havendo uma distribuição proporcional entre delineamentos transversais e longitudinais. Taylor (2010) sugere que para atender às questões que permeiam o desenvolvimento infantil, estudos longitudinais de base populacional são mais robustos. Tais limitações marcaram a análise dos estudos que compuseram essa revisão e, mesmo os que buscaram um acompanhamento longitudinal, por vezes o fizeram por período aquém do ideal para a observação das aquisições na totalidade de sua amostra (WALLE; CAMPOS, 2014).

A maioria dos estudos contemplaram análises de regressões (ALCOCK; KRAWCZYK, 2010; HE; WALLE; CAMPOS, 2015; OUDGENOEG-PAZ; VOLMAN; LESEMAN, 2012; WALLE; CAMPOS, 2014) e, nos outros estudos longitudinais incluídos (DARRAH et al., 2003; EJIRI; MASATAKA, 2001), a relação antecedente-consequente foi maciçamente pautada em análises de correlação. Conforme reforçado por Taylor (2010), a compreensão dos múltiplos fatores que influenciam o desenvolvimento infantil seria mais bem elucidada por análises de regressão logística multivariada, ajustada para efeitos preditores.

Para realizar tal análise, faz-se necessário elencar os potenciais facilitadores e os restritores, que podem interferir na aquisição de um determinado marco e ponderar por meio de modelagens a força de asso- 
ciação de cada uma dessas variáveis ante o desfecho analisado (D'SOUZA; D'SOUZA; KARMILOFF-SMITH, 2017). A heterogeneidade das variáveis analisadas nos estudos selecionados pode ter sido determinante para as conclusões diversas a que eles chegaram.

Apesar de a maioria dos estudos analisados nesta revisão apontarem uma associação entre processos de aquisição de algum marco motor com a aquisição da linguagem, estes não são conclusivos em unificar potenciais mecanismos envolvidos que possam justificar uma eventual interação. D'Souza, D'Souza e Karmiloff-Smith (2017), ao revisarem os precursores do desenvolvimento da linguagem em bebês e crianças em desenvolvimento típico e atípico, sugerem que essa análise deve ser conduzida sob a ótica da multifatorialidade, integrando múltiplas fontes de informação em contextos naturalistas, sem desconsiderar o tempo de desenvolvimento em um progresso cronológico de aquisições.

Não obstante de parecer uma sequência necessária e compreender o típico antes do atípico, os estudos mais encontrados nas bases de dados traçam uma interação entre os domínios na análise dos processos envolvidos em alterações na linguagem expressiva (CHOO et al., 2016; FREZZATO et al., 2017; LIU; ZAHRT; SIMMS, 2018). Nesta revisão, um dos estudos que não encontrou associação, ainda que em análise transversal, entre o componente motor amplo e a linguagem em uma amostra com desenvolvimento típico, sinaliza uma provável interação em caso de desordem (ALCOCK; KRAWCZYK, 2010). Segundo os autores, se habilidades motoras em idades precoces são preditoras de aquisições posteriores em linguagem, a avaliação de habilidades motoras auxiliaria na identificação precoce de crianças com comprometimento de fala e linguagem.

Os processos de aquisições motoras amplas, especialmente marcadas topograficamente por progressões céfalo-caudal e próximo-distal, mostram-se marcadores de uma maior autonomia funcional, exploratória e sensorial do ambiente que cerca o indivíduo em desenvolvimento. Esse aspecto passou a ficar mais claro nos três últimos revisados (HE; WALLE; CAMPOS, 2015; OUDGENOEG-PAZ; VOLMAN; LESEMAN, 2012; WALLE; CAMPOS, 2014), especialmente a partir de Oudgenoeg-Paz, Volman e Leseman (2012), que constataram, a partir de suas observações, que crianças que se sentaram sem apoio e caminharam independentemente antes, mostram um grau geral de linguagem mais alto e maior taxa de crescimento do vocabulário produtivo, respectivamente.
Os estudos subsequentes (HE; WALLE; CAMPOS, 2015; WALLE; CAMPOS, 2014) seguiram a mesma ótica ao observar a transição entre as formas de deslocamento independente, nesse caso do engatinhar para a caminhada. Walle e Campos (2014) observaram que a aquisição da caminhada esteve associada com um aumento significativo na linguagem receptiva e produtiva, independentemente da idade, ou seja, essa aquisição não seria unicamente dependente de um processo maturacional isolado. Eles discutem que seus achados sejam explicados por uma multifatorialidade que envolve mecanismos de desenvolvimento e maturação, interação entre habilidades verbais e motoras, bem como interação social e comunicativa do ambiente linguístico com seus pares.

Evoluindo na investigação dos fatores determinantes nessa interação, He, Walle e Campos (2015) apontam para a possibilidade de o marco motor ser um evento epigenético na aquisição da linguagem, e em seu estudo replicaram e ampliaram a amostra americana do estudo prévio de Walle e Campos (2014), incluindo crianças chinesas. Esse novo estudo teve a finalidade de analisar se a interação entre os domínios, encontrada no início da aquisição da marcha, seria uma resposta cultural ou estaria atrelada a um fenômeno epigenético. Os autores concluíram pela relação epigenética, pautada em uma mudança qualitativa do vocabulário, no desenvolvimento da linguagem associada ao desenvolvimento da marcha. Essa associação manifestou-se em culturas e idades distintas (crianças chinesas tendem a adquirir o marco motor até seis meses após a média da amostra americana), porém apresentaram incremento semelhante no vocabulário ao compararem crianças que engatinham e que passam a caminhar.

He, Walle e Campos (2015) também encontraram uma maior diferença em relação ao vocabulário expressivo associado à aquisição do marco motor, fator esse discutido enquanto dependente de uma resposta fisiológica postural necessária à produção da voz (diafragma/respiração) que pode ser influenciado pela locomoção vertical, sugerindo a necessidade de novas investigações que mais bem elucidem os fatores que permeiam essa relação complexa. Todas essas observações são reforçadas pelos estudos desenvolvidos por Iverson, desde a sua revisão de 2010 até estudos mais recentes que fundamentaram processos de aquisição típico e alterações que fortemente são impactadas pela restrição de experiências, como nos casos de transtorno do espectro do autismo (TEA) (IVERSON, 2018). 
Essa autora revisou teorias desde visões mais antigas que atribuíam paralelos na conquista de marcos motores e de linguagem à maturação neuromotora, as quais foram perdendo hegemonia desde a percepção de diferenças individuais: nas idades de aquisição; nas teorias emergentes a partir da década de 60 , que passaram a discutir mecanismos neurofisiológicos, associando especialmente gesto e fala; e, somando-se à compreensão dessa complexidade, nas teorias que analisam aspectos ambientais e comportamentais, quando novas habilidades motoras podem desempenhar um papel importante no desenvolvimento da fala, com as teorias de interações entre os domínios no processo de desenvolvimento (IVERSON, 2010).

Iverson (2018) reforça que a marcha, em comparação ao engatinhar, favoreceria o indivíduo com relação a alguns aspectos como interação com o meio, menor gasto energético, campo de visão (KRETCH; FRANCHAK; ADOLPH, 2014), liberação das mãos para a manipulação e exploração de objetos de interesse em uma interação social mais rica. Nesse sentido, os pais aumentariam as vocalizações para as crianças após a aquisição da marcha, facilitando o desenvolvimento linguístico das mesmas (KARASIK; TAMIS-LEMONDA; ADOLPH, 2014).

Mais recentemente, Iverson (2018) conceitua o desenvolvimento motor enquanto participante, mas nem lógico, nem causal, dos processos que envolvem a aquisição das habilidades comunicativas de linguagem. Essa relação de participação é determinante no desenvolvimento típico, pois ganhos na habilidade motora proporcionariam as vivências necessárias para potencializar o desenvolvimento da fala.

Numa descrição prática, a autora exemplifica situações que vão desde a liberação das mãos, quando o indivíduo passa a permanecer sentado sem apoio e pode manipular, explorar e ofertar objetos, dinamizando a interação com seus pares e evocando respostas dos mesmos, bem como um campo de visão diferenciado. Quando evolui para a locomoção independente, como o engatinhar, fica exposto a um meio com maiores oportunidades e experiências perceptivas e sociais que um cuidador atento a um novo padrão de movimentação pode proporcionar. Por fim, a deambulação consolida um complexo padrão postural que envolve desde capacidade respiratória e organização anátomo-fisiológica das estruturas fonadoras, até um maior controle dos segmentos apendiculares, visão e movimentação, resultando em grandes mudan- ças quantitativas e qualitativas na comunicação infantil, mencionando, inclusive, achados dos estudos aqui revisados.

O presente estudo objetivou identificar e sintetizar estudos observacionais que avaliaram ou descreveram algum processo de interação entre componentes do desenvolvimento motor amplo e da linguagem por meio da aquisição da fala, em um processo de desenvolvimento típico ainda na primeira infância. Algumas limitações impossibilitaram o desenvolvimento de um estudo mais robusto que possibilitasse uma maior precisão das relações envolvidas nesse processo complexo. Estas compreenderam desde a estruturação de uma questão de pesquisa que pudesse contemplar esse objetivo dentro dos critérios de uma revisão sistemática seguida de metanálise (objetivos bastante distintos, número amostral, rigor metodológico dos estudos, diversidade de avaliações), até uma carência de padronização de termos na literatura para unificar a busca.

O restrito número de estudos encontrados deve-se a uma especificidade de critérios que possibilita a compreensão dos mecanismos envolvidos no processo de aquisição típico, permitindo estabelecer uma relação clara e determinante para a deteç̧ão cada vez mais precoce do atípico. Essa escassez de informação também foi reportada por Ertem et al. (2018), sinalizando a importância fundamental desse conhecimento para a adequada promoção e monitoramento da saúde e do desenvolvimento infantil. Ainda assim, a metodologia escolhida cumpriu bem o papel de reunir achados relevantes para suscitar a revisão teórica necessária para encorajar a base metodológica de novos e promissores estudos envolvendo essa temática.

O desenvolvimento de novas habilidades no indivíduo típico parece estar cada vez mais amparado na multifatorialidade que determina a maioria dos eventos em saúde. Um processo maturacional, dado pelo avanço cronológico da idade, parece estruturar os alicerces que suportarão os aprendizados ditados por experiências em interação ambiental e social bidirecional, ou seja, o indivíduo manifesta habilidades para receber estímulo de seus pares, e esse estímulo é, também, fonte de aprendizado. Nesse complexo processo interacional, o desenvolvimento motor parece preparar terreno para o desenvolvimento da fala, favorecendo uma maior exploração do ambiente e gerando melhor resposta comunicativa, especialmente de pais e cuidadores.

Os estudos selecionados nesta revisão não oferecem um consenso que, provavelmente, pode ser explicado pela heterogeneidade metodológica, espe- 
cialmente distinguida pelo restrito número amostral, diversidade de objetivos, instrumentos e métodos de análise, notadamente marcada por a variável de desfecho ser centrada em escores gerais do domínio, ou marcos pontuais. Sugerem-se novos estudos observacionais que olhem para marcos específicos em interação, preferencialmente de modo longitudinal, para maior aproximação da relação antecedente consequente, envolvida no processo de desenvolvimento. Esses resultados, se analisados sob regressão, poderão estimar a força de associação de cada uma das variáveis que podem ser determinantes para o desfecho avaliado.

\section{REFERÊNCIAS}

ALCOCK, K. J.; KRAWCZYK, K. Individual differences in language development: Relationship with motor skill at 21 months. Developmental Science, v. 13, n. 5, p. 677-691, 2010.

CHOO, A. L. et al. Dissociations among linguistic, cognitive, and auditory-motor neuroanatomical domains in children who stutter. J Commun Disord., v. 61, p. 29-47, 2016.

D'SOUZA, D.; D'SOUZA, H.; KARMILOFF-SMITH, A. Precursors to language development in typically $\&$ atypically developing infants \& toddlers: The importance of embracing complexity. Journal of Child Language, v. 44, n. 3, p. 591627, 2017.

DARRAH, J. et al. Stability of serial assessments of motor and communication abilities in typically developing infants - Implications for screening. Early Human Development, v. 72, n. 2, p. 97-110, 2003.

EJIRI, K.; MASATAKA, N. Co-occurrence of preverbal vocal behavior and motor action in early infancy. Developmental Science, v. 4, n. 1, p. 40-48, 2001.

ERTEM, I. O. et al. Similarities and differences in child development from birth to age 3 years by sex and across four countries: a cross-sectional, observational study. The Lancet Global Health, v. 6, n. 3, p. e279-e291, 2018.

FREZZATO, R. C. et al. Fine motor skills and expressive language: a study with children with congenital hypotyreoidism. CoDAS, v. 29, n. 1, p. e20160064, 2017.

HE, M.; WALLE, E. A.; CAMPOS, J. J. A cross-national investigation of the relationship between infant walking and language development. Infancy, v. 20, n. 3, p. 283-305, 2015.

HODGSON, J. C.; HUDSON, J. M. Speech lateralization and motor control. Progress in Brain Research, v. 238, p. 145178, 2018.

IVERSON, J. M. Developing language in a developing body: the relationship between motor development and language development. Journal of Child Language, v. 37, n. 2, p. 229261, 2010.

IVERSON, J. M. Early motor and communicative development in infants with an older sibling with autism spectrum disorder. Journal of Speech, Language, and Hearing Research, v. 61, n. 11, p. 2.673-2.684, 2018.
KARASIK, L. B.; TAMIS-LEMONDA, C. S.; ADOLPH, K. E. Crawling and walking infants elicit different verbal responses from mothers. Developmental Science, v. 17, n. 3, p. 388395, 2014.

KOLB, B.; HARKER, A.; GIBB, R. Principles of plasticity in the developing brain. Developmental Medicine and Child Neurology, v. 59, n. 12, p. 1.218-1.223, 2017.

KRETCH, K. S.; FRANCHAK, J. M.; ADOLPH, K. E. Crawling and walking infants see the world differently. Child Development, v. 85, n. 4, p. 1.503-1.518, 2014.

LIU, X. L.; ZAHRT, D. M.; SIMMS, M. D. An Interprofessional Team Approach to the Differential Diagnosis of Children with Language Disorders. Pediatric Clinics of North America, v. 65, n. 1, p. 73-90, 2018.

LOGAN, S. W. et al. Fundamental motor skills: A systematic review of terminology. Journal of Sports Sciences, v. 36, n. 7, p. 781-796, 2018.

MANCINI, V. et al. Motor skills and internalizing problems throughout development: An integrative research review and update of the environmental stress hypothesis research. Research in Developmental Disabilities, v. 84, n. Aug. 2017, p. 96-111, 2019.

MEDINA ALVA, M. D. P. et al. Neurodesarrollo infantil: características normales y signos de alarma en el niño menor de cinco años. Rev Peru Med Exp Salud Publica, v. 32, n. 2, p. 565-573, 2015.

NELSON, E. L.; CAMPBELL, J. M.; MICHEL, G. F. Early handedness in infancy predicts language ability in toddlers. Developmental Psychology, v. 50, n. 3, p. 809-814, 2014.

OUDGENOEG-PAZ, O.; VOLMAN, M. C. J. M.; LESEMAN, P. P. $M$. Attainment of sitting and walking predicts development of productive vocabulary between ages 16 and 28 months. Infant Behavior and Development, v. 35, n. 4, p. 733-736, 2012.

SOUZA, M.; SILVA, M.; CARVALHO, R. Revisão integrativa: O que é? Como fazer isso? Einstein, São Paulo, v. 8, n. 1, p. 102-106, 2010.

TAYLOR, C. L. Early motor development is part of the resource mix for language acquisition. J Child Lang, v. 37, n. 2, p. 281-285, 2010.

VAINIO, L. Connection between movements of mouth and hand: Perspectives on development and evolution of speech. Neuroscience and Biobehavioral Reviews, v. 100, n. Nov. 2018, p. 211-223, 2019.

WALLE, E. A.; CAMPOS, J. J. Infant language development is related to the acquisition of walking. Developmental Psychology, v. 50, n. 2, p. 336-348, 2014.

ZAGO, J. T. C. et al. Associação entre o desenvolvimento neuropsicomotor e fatores de risco biológico e ambientais em crianças na primeira infância. Revista Cefac, v. 19, n. 3, p. 320-329, 2017. 